\title{
Motion speed modulates walking direction discrimination: The role of the feet in biological motion perception
}

\author{
CAI Peng ${ }^{1,2,3}$, YANG XiaoYing ${ }^{2,3}$, CHEN Lin $^{1 *} \&$ JIANG Yi ${ }^{2 *}$ \\ ${ }^{1}$ State Key Laboratory of Brain and Cognitive Science, Institute of Biophysics, Chinese Academy of Sciences, Beijing 100101, China; \\ ${ }^{2}$ Key Laboratory of Mental Health, Institute of Psychology, Chinese Academy of Sciences, Beijing 100101, China; \\ ${ }^{3}$ Graduate University of Chinese Academy of Sciences, Beijing 100049, China
}

Received March 5, 2011; accepted April 15, 2011

\begin{abstract}
The human visual system is tuned to the motions of biological entities, which provide potentially vital information for survival. The current study examines the interplay between motion speed and motion direction perception. Following a brief presentation of a point-light walker walking straight ahead or slightly leftward or rightward, observers were asked to quickly judge the walking direction (left or right). Participants showed better direction discrimination when the walker walked at a fast pace compared to a natural or slow pace, and this was not simply due to a difference in motion cycles. Moreover, walking direction sensitivity could be enhanced by increasing the feet motion speed alone, so long as the direction of feet movement was consistent with that of the other body parts. These findings demonstrate that our perception of walking direction is influenced by local motion speed, and highlight the role of the feet in biological motion perception.
\end{abstract}

biological motion, motion speed, walking direction, discrimination

Citation: $\quad$ Cai P, Yang X Y, Chen L, et al. Motion speed modulates walking direction discrimination: The role of the feet in biological motion perception. Chinese Sci Bull, 2011, 56: 2025-2030, doi: 10.1007/s11434-011-4528-6

Humans, like many other species, are fundamentally social and have evolved mechanisms that allow us to work successfully in large social groups. As social animals, humans behave largely in accordance with their interpretations and predictions about the actions of others, even in situations where visual information about a person is sparse, i.e. reduced to only a small number of point-lights attached to the head and major joints [1]. It has been demonstrated that observers can readily recognize the action [2,3], gender [4-8], and identity information [9-11] conveyed by point-light biological motion. Among these, detecting the direction of movement of an individual is crucial for a variety of adaptive behaviors including hunting and fleeing. Indeed, walking direction (left vs. right) can be successfully discriminated even when point-light displays are embedded in dynamic visual noise [12-16]. Young infants are able to

*Corresponding authors (email: linchen@bcslab.ibp.ac.cn; yijiang@psych.ac.cn) discriminate the walking direction of an upright point-light walker as early as 6 months old [17].

As people typically move forwards in the direction they are facing, walking direction is accordingly the same as their facing direction. In the case of the point-light walker stimuli used in these studies, the walkers walk on a treadmill and there is no global translational motion at all. Therefore, the walking direction is essentially equivalent to the facing direction. Previous studies have shown that the human visual system has specialized mechanisms tuned to different face viewpoints [18,19] and body orientations [20]. Although motion can provide more reliable and compelling direction information than a static frame [21], the role of the motion signals is still unclear, as well as whether local motion itself can influence viewpoint discrimination in biological motion. While many previous studies have focused on the global aspects of biological motion perception and have emphasized the contribution of the global 
configuration [13,22,23], some recent findings also provide evidence highlighting the unique role of local motion signals (e.g., the motion of the feet) in biological motion processing. It has been shown that the local features in biological motion can be processed independent of global configuration and influence the detection and visual search of biological motion signals [14,24-28]. In the current study, we further examined whether our perceptual discrimination of biological motion walking direction can be modulated by specific properties of the local motion signals.

Motion speed or velocity is one of the most important properties in characterizing common motion information over space and time. It has been shown that translational motion direction perception is affected by many factors including motion speed, stimulus contrast and duration. For humans and cats, the threshold of translation motion direction discrimination, measured by moving isotropic dot patterns, is inversely related to stimulus speed only at low drift rates $[29,30]$. As mentioned above, biological motion perception is quite different from translational motion in that the successful recognition and discrimination of biological motion is highly dependent on the global configural information [13,23,31-33] and might not necessarily rely on local motion signals. Here we aimed to first investigate whether and how motion speed modulates the discrimination of biological motion walking direction in the human visual system. In addition, we further examined the potential role of local motion signals in biological motion perception.

\section{Methods}

\subsection{Participants}

Fifty-two graduate students, who were between 19 and 30 years of age, participated in the current study (12 participants in Experiment 1, 14 in Experiment 2, 14 in Experiment 3, and 12 in Experiment 4). These participants did not overlap among the experiments. All observers had normal or corrected-to-normal vision and gave written informed consent in accordance with the procedures and protocols approved by the institutional review board of the Institute of Psychology, Chinese Academy of Sciences. They all perceived the point-light walker as facing towards themselves and were naïve to the purpose of the experiments.

\subsection{Stimuli and procedure}

Stimuli were generated and displayed using MATLAB (Mathworks, Inc.) together with the Psychophysics Toolbox extensions $[34,35]$. Point-light biological motion 3D coordinates were adopted from Vanrie and Verfaillie [36]. A set of point-light walker stimuli, consisting of a front direction and a number of side walking directions, were generated by projecting 3D models rotated in depth (clockwise or counter clockwise) onto a 2D plane. The testing stimuli included side walking directions, which had $\pm 2, \pm 4$, or \pm 6 degrees of angular rotation from the front direction, and the front direction itself. In Experiment 1, the point-light walker could walk at three different paces: fast $(2 \mathrm{cycle} / \mathrm{sec})$, natural (1 cycle/sec) and slow $(0.5 \mathrm{cycle} / \mathrm{sec})$ speeds. In Experiment 2, in addition to the motion speed, the number of motion cycles was also manipulated as to be either 1 or 2 cycles. In Experiment 3, only the motion speed of the two point-lights representing the feet was changed and all other point-lights remained at the natural speed. In Experiment 4, everything was the same as in Experiment 3 except that the moving direction of the feet was either kept normal or constant towards the front.

The stimuli were white on a grey background and the viewing distance was approximately $70 \mathrm{~cm}$. In each trial, the point-light biological motion stimulus $\left(4.3^{\circ} \times 10.7^{\circ}\right)$ appeared in the center of the screen with a small random displacement $\left(0^{\circ}-1^{\circ}\right)$ in order to avoid the potential interference effect from previous trials. Following the presentation of the stimulus, observers were required to report whether the walker was walking towards the left or the right side of the front direction by pressing one of two keys on a standard keyboard. There were 20 trials for each condition, distributed in four blocks, and all stimuli were presented in a truly randomized sequence. The participants of each experiment completed all blocks in one session. The data points from each participant for each walking speed condition were fit with a sigmoid Boltzmann function $\left(f(x)=1 /\left(1+\exp \left(\left(x-x_{0}\right) / \omega\right)\right)\right)$. Here the measurement of the discriminability of the biological motion walking direction was described as the difference limen (DL), which is estimated as being half the interquartile range of the fitted function $\left(\mathrm{DL}=x_{0.75}-x_{0.25}\right)$. In other words, the smaller the DL, the higher the discrimination sensitivity of the observer to the biological motion walking direction. In addition, the point of subjective equality (PSE) was taken to rate a walker walking towards the front. PSE and DL values were then subject to further group averaging and statistical tests.

\section{Results and discussion}

\subsection{Experiment 1: Motion speed modulates walking direction discrimination}

To examine the influence of the motion speed on the discrimination of walking direction, three different motion speeds (fast: 2 cycles/s; natural: 1 cycle/s; slow: 0.5 cycle/s) were tested in the experiment.

The results of Experiment 1 are plotted in Figure 1, which shows the mean PSE and DL values (see Methods for details). The DL was significantly modulated by the different motion speeds $(F(2,22)=12.57, P<0.001)$. Specifically, the DL under the fast walking speed $(2$ cycles/s) condition was significantly smaller than the DLs under the 
natural $(1$ cycle/sec; $t(11)=3.68, P<0.005)$ and the slow walking speed conditions $(0.5$ cycle/s; $t(11)=4.20, P<$ 0.001 ), and the difference of the DLs between the natural and slow walking speed conditions was not significant $(t(11)=0.79, P>0.4)$. In other words, observers demonstrated a better discrimination sensitivity when the point-light walker walked at a fast pace compared to when the walker walked at a natural or slow pace. The PSE was not different across the motion speed conditions $(F(2,22)=$ $0.18, P>0.7)$.

Although the presentation durations of the point-light walker were kept exactly the same under the different motion speed conditions, it could be argued that the number of motion cycles was not the same for each condition. For example, the point-light walker completed 2 motion cycles within 1 second under the fast walking speed condition (2 cycles/s), whereas only half of a cycle was displayed under the slow walking speed condition $(0.5 \mathrm{cycle} / \mathrm{s})$. It is possible that the number of motion cycles of biological motion, instead of the motion speed, is the key factor in modulating the discrimination sensitivity of the human visual system for perceiving walking direction of biological motion [15]. To test this possibility, Experiment 2 was designed so that both the motion speed and the motion cycle were manipulated.

\subsection{Experiment 2: The role of the motion cycle in walking direction discrimination}

In Experiment 2, each testing stimulus (point-light walker) could be presented with either a fast walking speed ( 2 cycles/s) or a natural walking speed (1 cycle/s), and could be presented for either one or two motion cycles. All other aspects of the procedure were the same as in Experiment 1.

The results of Experiment 2 are plotted in Figure 2. For PSE, repeated measures ANOVAs showed that neither the main effects of motion speed $(F(1,13)=2.81, P>0.1)$ and motion cycle $(F(1,13)=0.14, P>0.7)$ nor the interaction between motion speed and motion cycle $(F(1,13)=0.06, P$ $>0.8)$ were significant. These results were consistent with

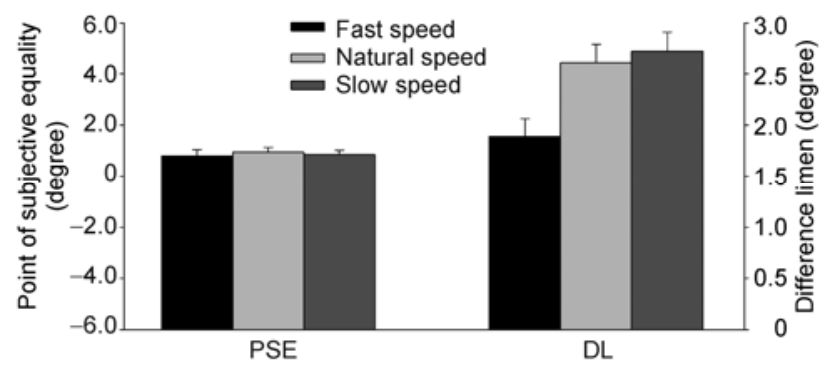

Figure 1 Results of Experiment 1 show the average PSE (point of subjective equality) and DL (difference limen) under different motion speed conditions across 12 participants. The PSE was not significantly different (left panel), but observers had better discrimination sensitivity when the point-light walker walked at a fast pace compared to a natural or slow pace (right panel). Error bars indicate one standard error.

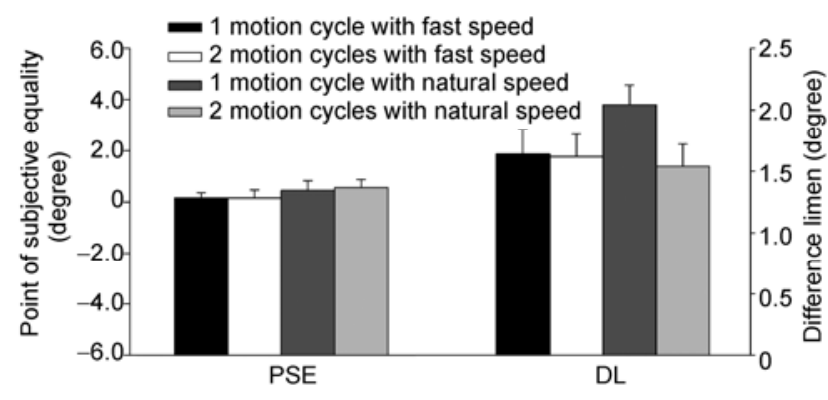

Figure 2 Results of Experiment 2 show the average PSE and DL across 14 participants. The PSE was not significantly different (left panel), but there was a significant interaction in DL (right panel). Error bars indicate one standard error.

those of Experiment 1, suggesting that PSE was not modulated by the motion speed or the motion cycle.

For DL, repeated measures ANOVAs showed that the main effects of motion speed $(F(1,13)=1.65, P>0.2)$ and motion cycle $(F(1,13)=2.61, P>0.1)$ were not significant, but there was a significant interaction between motion speed and motion cycle $(F(1,13)=6.97, P<0.05)$. Further tests of simple effects showed that when the presentation duration of the point-light walker was $1 \mathrm{~s}$ ( 2 cycles for fast walking speed and 1 cycle for natural walking speed), the DL under the fast walking speed condition was significantly smaller (indicating better discrimination) than the DL under the natural walking speed condition $(F(1,13)=6.50, P<0.05)$. This is basically a replication of the findings from Experiment 1 . Compared to the single motion cycle, the presentation of 2 motion cycles only reduced the DL under the natural walking speed condition $(F(1,13)=7.31, P<0.05)$, but did not have an effect under the fast walking speed condition $(F(1,13)=0.01, P>0.9)$. These results suggest that the number of motion cycles plays an important role in our discrimination of biological motion walking direction, consistent with previous findings [15]. However, the heightened discrimination performance obtained with fast-motionspeed stimuli could not be simply attributed to differences in the number of motion cycles. In fact, observers showed superior discrimination sensitivity for the fast walking walkers regardless of how many motion cycles were displayed. It is possible that the effect of number of motion cycles is limited to a certain range of biological motion walking speeds within which the observers' discrimination sensitivity increases with the number of cycles presented [15].

\subsection{Experiment 3: The role of feet in the modulation effect of motion speed}

The results from Experiments 1 and 2 provide clear evidence that motion speed modulates our perceived biological motion walking direction. Although the point-light walker is usually perceived as a global motion pattern, it is not clear whether the modulation effect arises from the global 
configuration or from the local motion signals. Recent studies have revealed that the motion of the feet, which move along longer trajectories than other points, contains meaningful biological properties and has a special role in biological motion perception [14,24-26]. To isolate the contribution of feet movement in the modulation effect observed here, we manipulated only the motion speed of the feet while keeping all other points unchanged.

The results of Experiment 3 are plotted in Figure 3. Paired-sample $t$-tests showed that, when compared with a single motion cycle, the presentation of 2 motion cycles reduced the DL under the natural walking speed condition $(t(13)=2.58, P<0.05)$, replicating the findings from Experiment 2. Critically, the DL with high feet motion speed was significantly smaller than the DL with natural feet motion speed, although the motion speed of all the other points was identical. Moreover, the independent-sample $t$-tests showed that the DL under the fast feet motion condition was similar to the DL under the fast walking speed condition that was observed in both Experiment $1(t(24)=0.07, P>$ $0.9)$ and Experiment $2(t(26)=1.18, P>0.2)$. These results indicate that, in terms of improving walking direction discrimination, there is no difference between increasing the motion speed of the point-lights representing the whole walker and increasing the motion speed of the feet only. Our data thus provides strong support for the notion that local feet motion plays a critical role in the visual discrimination of biological motion walking direction.

\subsection{Experiment 4: Modulation is not due to a general arousal effect}

The results from Experiment 3 reveal that biological motion walking direction discrimination can be modulated by local

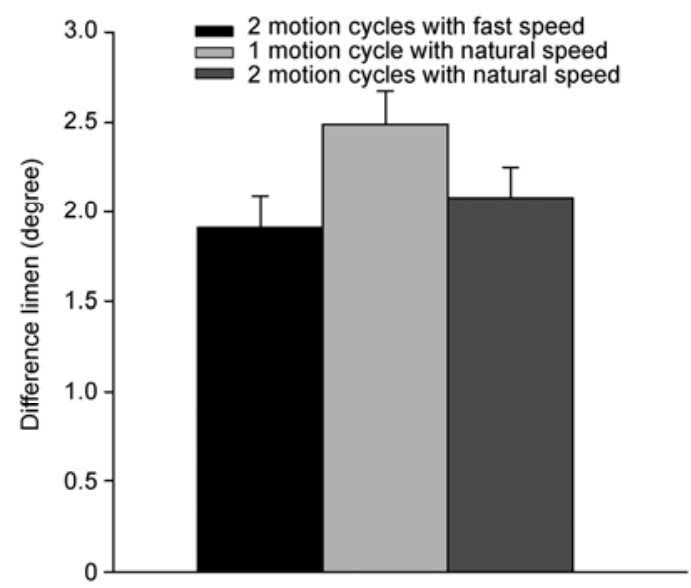

Figure 3 Results of Experiment 3 show the average DL across 14 participants. In order to isolate the contribution of the feet motion in the modulation effect observed from Experiments 1 and 2, only the motion speed of the feet was manipulated while all other points were unchanged (natural speed). Again, observers showed higher discrimination sensitivity for the fast feet motion stimulus. Error bars indicate one standard error. motion speed. However, it is possible that the modulation effect may simply reflect a general arousal effect rather than specific perceptual processing. In other words, the fast motion itself might have induced a general enhancement of arousal level, which could further mediate the modulation effect. If this is the case, we would expect that the modulation effect would be replicated even when the fast motion signal is not direction specific. To test this possibility, we manipulated the direction of the feet to be either consistent with the whole body (normal feet condition) or constantly walking forward (constant feet condition). In other words, under the normal feet condition, the stimuli were exactly the same as in Experiment 1; under the constant feet condition, the walking direction of the feet was constantly towards the front regardless of the facing direction of the other parts of the walker. All other variables were the same as in Experiment 3 .

The results of Experiment 4 (Figure 4) indicated that when feet direction was consistent with the rest of the body, the DL in the fast walking speed condition was significantly smaller than the DL in the natural walking speed condition $(t(11)=2.37, P<0.05)$, consistent with the results from Experiment 2. Importantly, when feet direction was kept constant and thus did not provide any useful walking direction information, the difference in the DL between the fast and natural walking speed conditions disappeared $(t(11)=$ $0.91, P>0.3$ ), indicating that the modulation effect of local motion speed was not due to a general arousal effect. Rather, it reflects a perceptual enhancement of discrimination sensitivity that is specific to the walking direction of the feet. Together, the current results reveal a significant role of feet in biological motion perception.

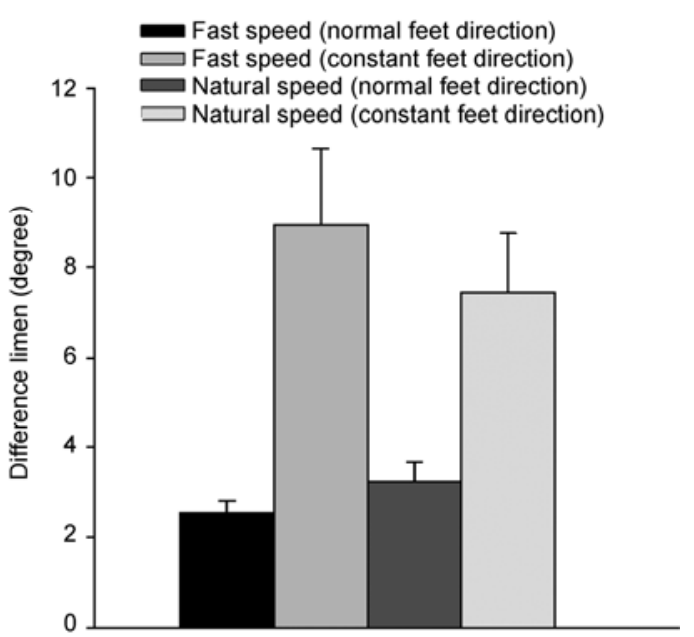

Figure 4 Results of Experiment 4 show the average DL across 12 participants. Normal feet direction means that the direction of the feet is consistent with the walking direction of the whole body, and constant feet direction means that the direction of the feet is constantly towards the front (zero degrees). The superior sensitivity for fast motion speed disappeared when the feet motion did not provide useful direction cues. Error bars indicate one standard error. 


\section{General discussion}

For a vast number of organisms, it is evolutionarily important to be highly sensitive to the moving direction of other biological entities under many different circumstances. Indeed, it has been demonstrated that the walking direction of point-light walkers can be incidentally processed [37] and even induce reflexive attentional orienting [21], suggesting an intrinsic sensitivity of the human visual attention system to biological motion signals [17,38,39]. Because a fast moving biological entity is more likely to signal a threat, it is thus crucial for an organisms survival to quickly and precisely judge its moving direction in order to make an appropriate response (e.g., fight-or-flight reaction). Because of this evolutionary salience, it is likely that such organism's would have a higher sensitivity to biological motion walking direction with higher motion speeds. In the current study, we consistently found that observers were more sensitive and performed better in discerning walking direction when the walker walked at a fast pace compared to a natural or slow pace. This result suggests that our perception of walking direction can be strongly modulated by the motion speed of biological motion. Although the number of motion cycles also has an impact on the discrimination sensitivity for biological motion walking direction [15], better discrimination for fast motion signals should not solely be attributed to the number of motion cycles in the different motion speed conditions.

Interestingly, walking direction sensitivity was enhanced by increasing the feet motion speed while keeping other variables unchanged. Critically, this enhancement disappeared when the motion direction of the feet was kept constant and thus did not provide any useful information about the walking direction. These results suggest that superior direction sensitivity for fast motion speed is not due to a general arousal effect but indeed reflects a perceptual enhancement of discrimination sensitivity that is specific to the walking direction of the feet. In other words, our perception of biological motion walking direction is strongly modulated by the local motion speed.

The current results are consistent with recent neuroimaging studies that have consistently identified the posterior superior temporal sulcus (pSTS) as being specifically sensitive to biological motion signals and playing a critical role in the perception of biological motion [40-44]. Because the pSTS receives both form analysis from the ventral stream and image motion signals from the dorsal stream, it is possible that biological motion perception can be influenced by both form and motion signals. On one hand, we have demonstrated a significant modulation effect of local motion speed. On the other hand, the form information (e.g., normal feet condition vs. constant feet condition in Experiment 4) affected the observers' judgment about walking direction.
Taken together, our findings suggest that our sensitivity to biological motion walking direction can be strongly modulated by the motion speed, and that such modulation critically depends upon the motion of the feet. These results highlight the role of local motion signals in biological motion walking direction discrimination, and provide new insights into the mechanisms underlying biological motion perception.

This work was supported by the National Basic Research Program of China (2011CB711000), the Knowledge Innovation Program of Chinese Academy of Sciences (09CX202020), and the National Natural Science Foundation of China (30921064, 31070903 and 90820307).

1 Johansso G. Visual-perception of biological motion and a model for its analysis. Percept Psychophys, 1973, 14: 201-211

2 Dittrich W H. Action categories and the perception of biological motion. Perception, 1993, 22: 15-22

3 Norman J F, Payton S M, Long J R, et al. Aging and the perception of biological motion. Psychol Aging, 2004, 19: 219-225

4 Kozlowski L T, Cutting J E. Recognizing the sex of a walker from a dynamic point light display. Percept Psychophys, 1977, 21: 575-580

5 Kozlowski L T, Cutting J E. Recognizing the gender of walkers from point-lights mounted on ankles: Some second thoughts. Percept Psychophys, 1978, 23: 459

6 Mather G, Murdoch L. Gender discrimination in biological motion displays based on dynamic cues. Proc R Soc Lond B Biol Sci, 1994, 258: 273-279

7 Sumi S. Perception of point-light walker produced by eight lights attached to the back of the walker. Swiss J Psychol, 2000, 59: 126-132

8 Troje N F. Decomposing biological motion: A framework for analysis and synthesis of human gait patterns. J Vis, 2002, 2: 371-387

9 Cutting J E, Kozlowski L T. Recognition of friends by their walk: Gait perception without familiarity cues. Bull Psychon Soc, 1977, 9: 353-356

10 Loula F, Prasad S, Harber K, et al. Recognizing people from their movements. J Exp Psychol Hum Percept Perform, 2005, 31: 210-220

11 Troje N F, Westhoff C, Lavrov M. Person identification from biological motion: Effects of structural and kinematic cues. Percept Psychophys, 2005, 67: 667-675

12 Aaen-Stockdale C, Thompson B, Hess R F, et al. Biological motion perception is cue-invariant. $\mathrm{J}$ Vis, 2008, 8: 1-11

13 Bertenthal B I, Pinto J. Global processing of biological motions. Psychol Sci, 1994, 5: 221-225

14 Mather G, Radford K, West S. Low-level visual processing of biological motion. Proc R Soc Lond B Biol Sci, 1992, 249: 149-155

15 Neri P, Morrone M C, Burr D C. Seeing biological motion. Nature, 1998, 395: 894-896

16 Thurman S M, Grossman E D. Temporal "Bubbles" reveal key features for point-light biological motion perception. J Vis, 2008, 8: 28. $1-11$

17 Kuhlmeier V A, Troje N F, Lee V. Young infants detect the direction of biological motion in point-light displays. Infancy, 2010, 15: 83-93

18 Fang F, Ijichi K, He S. Transfer of the face viewpoint aftereffect from adaptation to different and inverted faces. J Vis, 2007, 7: 6. 1-9

19 Fang F, He S. Viewer-centered object representation in the human visual system revealed by viewpoint aftereffects. Neuron, 2005, 45: 793-800

20 Lawson R P, Clifford C W G, Calder A J. About turn: The visual representation of human body orientation revealed by adaptation. Psychol Sci, 2009, 20: 363-371

21 Shi J, Weng X, He S, et al. Biological motion cues trigger reflexive attentional orienting. Cognition, 2010, 117: 348-354

22 Pinto J, Shiffrar M. Subconfigurations of the human form in the 
perception of biological motion displays. Acta Psychol, 1999, 102: 293-318

23 Shiffrar M, Lichtey L, Chatterjee S H. The perception of biological motion across apertures. Percept Psychophys, 1997, 59: 51-59

24 Chang D H, Troje N F. Characterizing global and local mechanisms in biological motion perception. J Vis, 2009, 9: 8. 1-10

25 Saunders D R, Suchan J, Troje N F. Off on the wrong foot: Local features in biological motion. Perception, 2009, 38: 522-532

26 Troje N F, Westhoff C. The inversion effect in biological motion perception: Evidence for a "life detector"? Curr Biol, 2006, 16: $821-824$

27 Wang L, Zhang K, He S, et al. Searching for life motion signals. Visual search asymmetry in local but not global biological-motion processing. Psychol Sci, 2010, 21: 1083-1089

28 Troje N F. Decomposing biological motion: A framework for analysis and synthesis of human gait patterns. J Vis, 2002, 2: 371-387

29 Debruyn B, Orban G A. Human velocity and direction discrimination measured with random dot patterns. Vis Res, 1988, 28: 1323-1335

30 Pasternak T, Merigan W H. Effects of stimulus speed on direction discriminations. Vis Res, 1984, 24: 1349-1355

31 Beintema J A, Lappe M. Perception of biological motion without local image motion. Proc Natl Acad Sci USA, 2002, 99: 5661-5663

32 Chatterjee S H, Freyd J J, Shiffrar M. Configural processing in the perception of apparent biological motion. J Exp Psychol Hum Percept Perform, 1996, 22: 916-929

33 Lange J, Lappe M. A model of biological motion perception from configural form cues. J Neurosci, 2006, 26: 2894-2906
34 Brainard D H. The psychophysics toolbox. Spat Vis, 1997, 10: 433436

35 Pelli D G. The VideoToolbox software for visual psychophysics: Transforming numbers into movies. Spat Vis, 1997, 10: 437-442

36 Vanrie J, Verfaillie K. Perception of biological motion: A stimulus set of human point-light actions. Behav Res Method Instrum Comput, 2004, 36: 625-629

37 Thornton I M, Vuong Q C. Incidental processing of biological motion. Curr Biol, 2004, 14: 1084-1089

38 Simion F, Regolin L, Bulf H. A predisposition for biological motion in the newborn baby. Proc Natl Acad Sci USA, 2008, 105: 809-813

39 Vallortigara G, Regolin L, Marconato F. Visually inexperienced chicks exhibit spontaneous preference for biological motion patterns. PLoS Biol, 2005, 3: 1312-1316

40 Saygin A P, Wilson S M, Hagler D J Jr, et al. Point-light biological motion perception activates human premotor cortex. J Neurosci, 2004, 24: 6181-6188

41 Thompson J C, Clarke M, Stewart T, et al. Configural processing of biological motion in human superior temporal sulcus. J Neurosci, 2005, 25: 9059-9066

42 Grossman E D, Blake R. Brain areas active during visual perception of biological motion. Neuron, 2002, 35: 1167-1175

43 Vaina L M, Solomon J, Chowdhury S, et al. Functional neuroanatomy of biological motion perception in humans. Proc Natl Acad Sci USA, 2001, 98: 11656-11661

44 Grossman E D, Blake R. Brain areas involved in perception of biological motion. J Cogn Neurosci, 2000, 12: 711-720

Open Access This article is distributed under the terms of the Creative Commons Attribution License which permits any use, distribution, and reproduction in any medium, provided the original author(s) and source are credited. 